TRANSACTIONS OF THE

AMERICAN MATHEMATICAL SOCIETY

Volume 363, Number 7, July 2011, Pages 3553-3572

S 0002-9947(2011)05114-7

Article electronically published on February 18, 2011

\title{
NON-GENERIC CUSPS
}

\author{
MICHA£ MISIUREWICZ AND ANA RODRIGUES
}

\begin{abstract}
We find the order of contact of the boundaries of the cusp for twoparameter families of vector fields on the real line or diffeomorphisms of the real line, for cusp bifurcations of codimensions 1 and 2. Moreover, we create a machinery that can be used for the same problem in higher codimensions and perhaps for other, similar problems.
\end{abstract}

\section{INTRODUCTION}

In the study of two-parameter families of circle maps, one encounters various objects that can be called tongues or cusps. The most popular are classical Arnold tongues. They are sets of parameters for which there is an attracting periodic point of a given rotation number. They can be encountered whenever in a more complicated system one gets an invariant circle on which the map is a homeomorphism, and the rotation number of this homeomorphism varies with the parameters. They have been studied extensively by various authors; see for instance $2,3,4,4,6,6,10$, and other papers cited there.

A standard example of a family in which Arnold tongues appear is the family of standard maps

$$
A_{a, b}(x)=x+a+\frac{b}{2 \pi} \sin (2 \pi x)(\bmod 1)
$$

introduced by Arnold in [1]. In [8, 9] we studied the family of double standard maps, which are obtained from the standard maps by replacing rotations of the circle by the composition of its doubling with rotations:

$$
f_{a, b}(x)=2 x+a+\frac{b}{\pi} \sin (2 \pi x)(\bmod 1) .
$$

In this family, tongues (sets of parameter values for which there is an attracting periodic point, this time of a given type (see [8]), not a given rotation number) also appear. However, their nature is different, since here for the values of the parameters at the tip of a tongue the system undergoes the cusp bifurcation (see [7]), which is not the case for the Arnold tongues. Thus, perhaps a more proper name for them is cusps.

Received by the editors December 14, 2008 and, in revised form, April 23, 2009.

2010 Mathematics Subject Classification. Primary 37G15, 37E99.

Key words and phrases. Cusp bifurcation, tongues, order of contact.

The first author was partially supported by NSF grant DMS 0456526 .

The second author was supported by FCT Grant BPD/36072/2007. Research of the second author was supported in part by Centro de Matemática da Universidade do Porto (CMUP) financed by FCT through the programmes POCTI and POSI, with Portuguese and European Community structural funds.

(C)2011 American Mathematical Society Reverts to public domain 28 years from publication 
Once the existence of cusps and their order are established, the next natural problem is about their shapes. One of the basic characteristics of the shape is the order of contact of the left and the right boundaries of the cusp at its tip. If those boundaries are of the form $a=B_{1}(b)$ and $a=B_{2}(b)$ (here the $a$-axis is horizontal and the $b$-axis vertical) and the tip of the cusp is at $\left(a_{0}, b_{0}\right)$, then this order is $r$ if the limit

$$
\lim _{b \rightarrow b_{0}} \frac{\left|B_{1}(b)-B_{2}(b)\right|}{\left|b-b_{0}\right|^{r+1}}
$$

is positive and finite. In other words, the order of contact is $r$ if the width of the cusp decreases to 0 as $\left|b-b_{0}\right|^{r+1}$ times a constant. For Arnold tongues in the family of standard maps the order of contact depends on the rotation number of the tongue. If the rotation number is $p / q$ (with $p$ and $q$ coprime), then the order is $q-1$ (see [1]). For the family of double standard maps the situation is different. By the results for the cusp bifurcation (see, e.g., [7]), the generic order of contact is $1 / 2$. In 8 we checked that this is the order for the cusp corresponding to the attracting fixed point. However, we do not know whether the situation is generic for tips of cusps corresponding to the attracting periodic points of higher period. This motivates the study of the order of contact at the cusp bifurcation point for non-generic cases, which we perform here.

In fact, this problem is interesting by itself, and since our setup has nothing to do with the specific situation of double standard maps, the results can be applied to much more general cases.

The aim of this paper is twofold. We find the order of contact of the boundaries of the cusp for two-parameter families of vector fields on the real line or diffeomorphisms of the real line, in codimensions 1 and 2 (we also include the generic case for completeness). What is perhaps more important, we create a machinery that can be used for the same problem in higher codimensions (although there will be more and more cases) and perhaps for other, similar problems.

As we said, we consider a two-parameter family of vector fields on the real line or diffeomorphisms of the real line. We look at them locally, so both parameters $a, b$ and the variable $x$ will be taken from some neighborhoods of 0 . Thus, we will work in a neighborhood $U$ of $(0,0,0)$ in $\mathbb{R}^{3}$. In order to have all continuous functions bounded automatically, we assume that $U$ is compact. We will be analyzing the shape of the bifurcation set in the cusp bifurcation - generic and of small codimensions.

The bifurcation set in the cusp bifurcation is given as the projection to the $(a, b)$-plane of the set of all solutions to the system of equations:

$$
\left\{\begin{array}{l}
F(a, b, x)=0, \\
F_{x}(a, b, x)=0 .
\end{array}\right.
$$

Here $F$ is the vector field in the continuous case and is the map minus the identity in the discrete case. For simplicity, we will assume that $F$ is real analytic. However, it is enough to assume that it is sufficiently smooth, that is, that all derivatives used in the formulas and proofs exist and are continuous.

We will denote by $T$ the set of solutions to (1.1), that is,

$$
T=\left\{(a, b, x) \in U: F(a, b, x)=0, \quad F_{x}(a, b, x)=0\right\} .
$$


In order to make (1.1) relevant for the cusp bifurcation, we write $F(0,0, x)=f(x)$ and assume that there is an odd integer $k \geq 3$ such that

$$
f(0)=f^{\prime}(0)=f^{\prime \prime}(0)=\cdots=f^{(k-1)}(0)=0, \quad f^{(k)}(0) \neq 0 .
$$

If we write $F$ in the form

$$
F(a, b, x)=A_{0}(a, b)+A_{1}(a, b) x+A_{2}(a, b) x^{2}+\cdots+A_{k}(a, b) x^{k}+\varphi x^{k+1},
$$

where $\varphi$ is an analytic (or sufficiently smooth) function, we have

$$
A_{0}(0,0)=A_{1}(0,0)=\cdots=A_{k-1}(0,0)=0, \quad A_{k}(0,0) \neq 0 .
$$

Consider the gradients $V_{0}$ of $A_{0}$ at the origin and $V_{1}$ of $A_{1}$ at the origin. Generically, for the cusp bifurcation, $k=3$, and $V_{0}$ and $V_{1}$ are linearly independent. We increase the codimension by increasing the number of equalities in the assumptions. Observe that $k$ in (1.4) has to be odd, and replacing $k=3$ by $k=5$ requires adding two extra equalities. Thus, the only possibility to get codimension 1 is to assume that $V_{0}$ and $V_{1}$ are non-zero, linearly dependent, and $k=3$ (a gradient is a vector, so to make it zero we need two equalities). In the study of this case in Section 4 we come across certain scalar quantities, $\beta$ and $\Delta$, which we can assume to be non-zero without changing the codimension. In particular, the results will depend on the sign of $\Delta$. In codimension 2 we have four possibilities. We can assume that $k=5$, that $V_{0}$ is zero, that $\Delta=0$, or that $\beta=0$. Assuming that $V_{1}$ is zero does not give us anything new, since in the codimension 1 case we do not assume that it is non-zero.

In order to summarize the results, we produce a table. In Table 1 we indicate the codimension, the number $k$, information about $V_{0}$ and $V_{1}$, and about $\beta$ and $\Delta$ (if relevant). Then we state what kind of cusp we get. It can be simple, consisting of two curves beginning at the origin and tangent there, or double, consisting of two curves passing through the origin and tangent there (see Figure 1). In those cases we also give the order of contact of those curves. However, we can also get a non-cusp, where the two curves begin at the origin, but the angle between them is $\pi$ (see also Figure 1), or we can even get the origin isolated (no curves nearby).
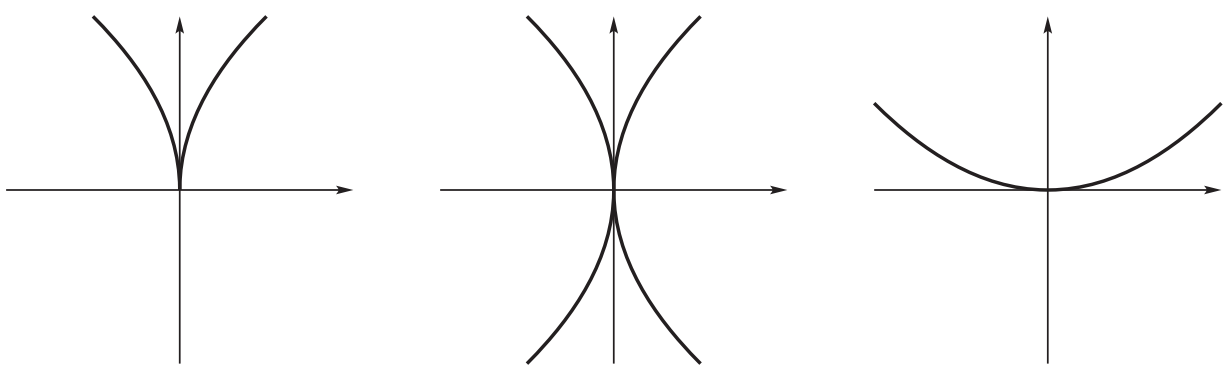

Figure 1. From the left: a simple cusp, a double cusp, and a non-cusp.

The paper is organized as follows. In Section 2 we present the main idea of the proofs and derive the main technical tool of the paper, which will be used in all other sections, except the last one. In Section 3 we study the well-known generic case for the sake of completeness and in order to illustrate our methods in a relatively simple case. Moreover, there we treat all $k \geq 3$ odd, which in particular covers 
TABLE 1. Summary of the results.

\begin{tabular}{|c|c|c|c|c|}
\hline Codimension & & Assumptions & Type of cusp & $\begin{array}{l}\text { Order of } \\
\text { contact }\end{array}$ \\
\hline 0 & $k=3$ & $V_{0}$ and $V_{1}$ linearly independent & simple cusp & $1 / 2$ \\
\hline 1 & $k=3$ & $\begin{array}{l}V_{0} \text { and } V_{1} \text { linearly dependent, } \\
V_{0} \text { non-zero, } \beta \neq 0, \Delta>0\end{array}$ & double cusp & 2 \\
\hline 1 & $k=3$ & $\begin{array}{l}V_{0} \text { and } V_{1} \text { linearly dependent, } \\
V_{0} \text { non-zero, } \beta \neq 0, \Delta<0\end{array}$ & isolated point & \\
\hline 2 & $k=5$ & $V_{0}$ and $V_{1}$ linearly independent & simple cusp & $1 / 4$ \\
\hline 2 & $k=3$ & $\begin{array}{l}V_{0} \text { and } V_{1} \text { linearly dependent, } \\
V_{0} \text { non-zero, } \beta \neq 0, \Delta=0\end{array}$ & simple cusp & $7 / 2$ \\
\hline 2 & $k=3$ & $V_{0}$ zero, $V_{1}$ non-zero & non-cusp & \\
\hline 2 & $k=3$ & $\begin{array}{l}V_{0} \text { and } V_{1} \text { linearly dependent, } \\
V_{0} \text { non-zero, } \beta=0, \Delta \neq 0\end{array}$ & double cusp & 2 \\
\hline
\end{tabular}

one case of codimension 2. In Section 4 we study the codimension 1 case, and in Sections 5, 6 and 7 all remaining codimension 2 cases.

\section{MAin Lemma}

Suppose that we have the system of equations

$$
\left\{\begin{array}{l}
a \mu(a, b, x)+\sigma(b, x)=0, \\
a \nu(a, b, x)+\tau(b, x)=0,
\end{array}\right.
$$

where $\mu(0,0,0)=1, \tau(b, x)=\sum_{i, j} c_{i j} b^{i} x^{j}, \sigma(b, x)=\sum_{i, j} d_{i j} b^{i} x^{j}$ and $\nu(a, b, x)=$ $\sum_{l, i, j} e_{l i j} a^{l} b^{i} x^{j}$. Choose the smallest $p$ such that $c_{0 p} \neq 0$ and the smallest $q$ such that $c_{q 0} \neq 0$. Assume that

(0) $\mu(0,0,0)=1$ 
and there is $t>0$ such that:

(1) if $\frac{i}{q}+\frac{j}{p}<1$, then $c_{i j}=0$,

(2) if $\frac{i}{q}+\frac{j}{p}<t$, then $d_{i j}=0$,

(3) if $\frac{i}{q}+\frac{j}{p}+t+t l<1$, then $e_{l i j}=0$,

(4) if $q t$ is an integer, then

$$
\sum_{\substack{l, i \\ i / q+t+t l=1}} e_{l i 0}\left(-d_{q t, 0}\right)^{1+l}+c_{q 0} \neq 0,
$$

(5) if $p t$ is an integer, then

$$
\sum_{\substack{l, j \\ j / p+t+t l=1}} e_{l 0 j}\left(-d_{0, p t}\right)^{1+l}+c_{0 p} \neq 0 .
$$

In concrete cases we will illustrate assumptions (1) and (2) in figures. We will use a grid to indicate the lowest order non-zero coefficients (with respect to $b$ and $x$ ) of $\sigma$ and $\tau$. We use circles for $\sigma$ and dots for $\tau$. The choice of $p$ and $q$ is indicated by the dots on the $x$ and $b$ axes, respectively. In Figure 2 we have $p=2$ and $q=1$.

Condition (1) means that there are no dots to the left of the line $L$ drawn through $(0, p)$ and $(q, 0)$. Condition $(2)$ means that if we draw the line $L^{\prime}$ through $(0, t p)$ and $(t q, 0)$, then there are no circles to the left of $L^{\prime}$. In Figure 2 we have $t=3 / 2$.

Our main idea is that in order for a function to be zero on some set close to the origin, we must have at least two non-zero terms of the lowest order, so they can cancel each other. The dots represent those terms, and the slope of the line depends on the relative order of the variables $x$ and $b$ on $T$.

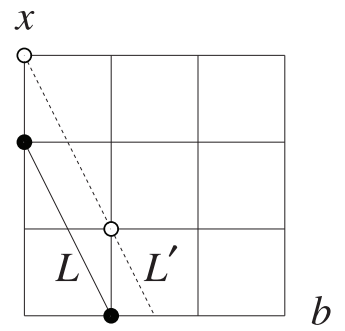

Figure 2. An example.

Lemma 2.1. Under the hypotheses (0)-(5), there exist constants $K_{1}, K_{2}, K_{3}$ and $K_{4}$ such that $|b|^{q} \leq K_{1}|x|^{p},|x|^{p} \leq K_{2}|b|^{q},|a| \leq K_{3}|b|^{t q}$ and $|a| \leq K_{4}|x|^{t p}$ in a small neighborhood of $(0,0,0)$ in the set $T$ of solutions to (2.1).

Proof. We want to prove that there exists a constant $K_{1}$ such that $|b|^{q} /|x|^{p} \leq K_{1}$ in a small neighborhood of $(0,0,0)$ in $T$. Suppose that this is not the case. Then there exists a sequence of points $\left(a_{n}, b_{n}, x_{n}\right) \in T$ convergent to $(0,0,0)$ such that $x_{n}^{p} / b_{n}^{q} \rightarrow 0$. 
Set $z=x^{p} / b^{q}$ in (2.1) and divide the first equation by $b^{t q}$ and the second equation by $b^{q}$ :

$$
\begin{aligned}
\frac{a}{b^{t q}} \mu\left(a, b,\left(z b^{q}\right)^{1 / p}\right)+\sum_{i, j} d_{i j} b^{q\left(\frac{i}{q}+\frac{j}{p}-t\right)} z^{j / p} & =0, \\
\frac{a}{b^{q}} \nu\left(a, b,\left(z b^{q}\right)^{1 / p}\right)+\sum_{i, j} c_{i j} b^{q\left(\frac{i}{q}+\frac{j}{p}-1\right)} z^{j / p} & =0 .
\end{aligned}
$$

In the sums, by assumptions (1) and (2), the powers are non-negative. The above formulas hold in particular for the sequence $\left(a_{n}, b_{n}, z_{n}\right)$. Consider the limit of the left hand side of (2.2) as $n \rightarrow \infty$. In the first expression we take (0) into account. In the sum, since $z_{n} \rightarrow 0$, we are left with the terms with $j=0$. Also $b_{n} \rightarrow 0$, so we are left with terms with $i / q-t=0$. Thus, we get

$$
\lim _{n \rightarrow \infty} \frac{a_{n}}{b_{n}^{t q}}=-d_{q t, 0}
$$

provided $q t$ is an integer; otherwise the limit is zero. Similarly, for the sum appearing in (2.3), in view of (1) we get

$$
\lim _{n \rightarrow \infty} \sum_{i, j} c_{i j} b_{n}^{q\left(\frac{i}{q}+\frac{j}{p}-1\right)} z_{n}^{j / p}=c_{q 0} .
$$

We now compute the limit of the left hand side of (2.3) for $\left(a_{n}, b_{n}, z_{n}\right)$ as $n \rightarrow \infty$. Set $w_{n}=\frac{a_{n}}{b_{n}^{t q}}$, so $a_{n}=w_{n} b_{n}^{t q}$. Then

$$
\frac{a_{n}}{b_{n}^{q}} \nu\left(a_{n}, b_{n},\left(z_{n} b_{n}^{q}\right)^{1 / p}\right)=\sum_{l, i, j} e_{l i j} w_{n}^{1+l} z_{n}^{j / p} b_{n}^{q\left(\frac{i}{q}+\frac{j}{p}+t+t l-1\right)} .
$$

In the limit when $n \rightarrow \infty$, the only possible non-zero terms in the above sum will be when $j=0$ and $i / q+t+t l=1$. Therefore, taking (2.5) into account, we get

$$
\lim _{n \rightarrow \infty} \sum_{\substack{l, i \\ i / q+t+t l=1}} e_{l i 0} w_{n}^{1+l}+c_{q 0}=0,
$$

where the limit of $w_{n}$ is given by (2.4). We take the sum over those $l, i$ for which $i / q+t+t l=1$. There are finitely many such pairs, and we get

$$
\sum_{\substack{l, i \\ i / q+t+t l=1}} e_{l i 0}\left(-d_{q t, 0}\right)^{1+l}+c_{q 0}=0 .
$$

This contradicts (4) if $q t$ is an integer. If $q t$ is not an integer, we get $c_{q 0}=0$, contrary to the choice of $q$. This proves the existence of $K_{1}$ such that $|b|^{q} /|x|^{p} \leq K_{1}$.

We use the same argument with the following pairs switched: $b, x ; p, q ; i, j$; $c_{q 0}, c_{0 p} ; d_{q t, 0}, d_{0, p t}$. Assuming (5) we prove that there exists a constant $K_{2}$ such that $|x|^{p} \leq K_{2}|b|^{q}$ in a small neighborhood of $(0,0,0)$ in $T$.

The inequality $|a| \leq K_{3}|b|^{t q}$ follows immediately from (2.2), since $b$ and $z$ are bounded and the sum in (2.2) is a continuous function of $b$ and $z$. Again we switch $b$ with $x$ and $p$ with $q$ to get $|a| \leq K_{4}|x|^{t p}$. This completes the proof. 


\section{GENERIC CASE}

The generic case is well known; see for instance [7]. However, we include it here to show in the simplest case how our method is working. Moreover, we generalize it a bit. Namely, in the generic case we have $k=3$. However, since $k=5$ appears in the codimension 2 case and the proofs are the same for all odd $k \geq 3$, here we will treat a more general situation with any value of $k \geq 3$ odd.

By $\varphi_{1}, \varphi_{2}, \ldots$ we will denote analytic (or sufficiently smooth) functions of the variables $a, b, x$. We will omit their arguments, since usually the only important property of those functions is that they are bounded in $U$ (and occasionally we will have to differentiate them, but not explicitly).

We write $F$ in the form (1.3) with conditions (1.4). The first generic assumption is that the gradient of $A_{0}$ at the origin is non-zero. Therefore we can change (diffeomorphically) coordinates in the parameter plane and assume that

$$
A_{0}(a, b) \equiv a
$$

(actually, we can also assume that $A_{1}(a, b) \equiv b$, but we do not need it). Therefore (1.3) takes the form

$$
F(a, b, x)=a+A_{1}(a, b) x+A_{2}(a, b) x^{2}+\cdots+A_{k}(a, b) x^{k}+\varphi x^{k+1} .
$$

Another generic assumption that we make is that the gradients of $A_{0}$ and $A_{1}$ at the origin are linearly independent. In view of (3.1), this assumption means that

$$
\frac{\partial A_{1}}{\partial b}(0,0) \neq 0 \text {. }
$$

By (1.4) and (3.3), those numbers are non-zero. Now, taking into account (1.4), we can write

$$
\begin{aligned}
F(a, b, x) & =a+\varepsilon b x+\delta x^{k}+\varphi_{1} a x+\varphi_{2} b^{2} x+\varphi_{3} b x^{2}+\varphi_{4} x^{k+1}, \\
F_{x}(a, b, x) & =\varepsilon b+k \delta x^{k-1}+\varphi_{5} a+\varphi_{6} b^{2}+\varphi_{7} b x+\varphi_{2} x^{k}
\end{aligned}
$$

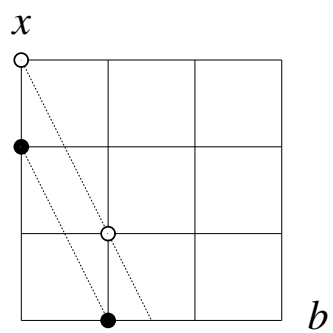

Figure 3. The lowest order non-zero coefficients for equations (3.5) and (3.6) with $k=3$.

Lemma 3.1. There exist constants $K_{1}, K_{2}, K_{3}, K_{4}$ such that $|b| \leq K_{1}|x|^{k-1},|x|^{k-1}$ $\leq K_{2}|b|,|a| \leq K_{3}|b|^{k /(k-1)}$ and $|a| \leq K_{4}|x|^{k}$. 
Proof. We claim that assumptions (0)-(5) of Lemma 2.1 are satisfied with $p=k-1$, $q=1$ and $t=\frac{k}{k-1}$. Indeed, $\mu(0,0,0)=1$, so (0) holds. Next, $\tau(b, x)=\varepsilon b+k \delta x^{k-1}+$ $\cdots$, so $c_{10}=\varepsilon \neq 0, c_{0, k-1}=k \delta \neq 0$ and $c_{00}=c_{01}=\cdots=c_{0, k-2}=0$; see the dots in Figure 3. Also $\sigma(b, x)=\varepsilon b x+\delta x^{k}+\cdots$, so $d_{11}=\varepsilon \neq 0, d_{0 k}=\delta \neq 0$ and $d_{00}=d_{01}=\cdots=d_{0, k-1}=d_{10}=0$; see the circles in Figure 3 (the only pairs $(i, j)$ for which $i+j /(k-1)<k /(k-1)$ are $(0,0),(0,1), \ldots,(0, k-1)$ and $(1,0))$. This proves (1) and (2). Since $t \geq 1$, there are no $(l, i, j)$ satisfying the inequality in (3), so (3) holds. The number $q t$ is not an integer, so (4) holds. Furthermore, $p t=k$ is an integer, but since $t>1$, the sum in (5) is empty. Thus, since $c_{0 p}=k \delta \neq 0,(5)$ holds. Therefore, we can use Lemma 2.1, and the lemma follows.

Lemma 3.2. As $(a, b, x) \in T$ and $x \rightarrow 0$, then $b / x^{k-1} \rightarrow-k \delta / \varepsilon$ and $a / x^{k} \rightarrow$ $(k-1) \delta$.

Proof. By Lemma 3.1 the last 4 terms in (3.6) are bounded by $|x|^{k}$ times a constant independent of $a, b, x$. This proves that $b / x^{k-1} \rightarrow-k \delta / \varepsilon$ as $(a, b, x) \in T$ and $x \rightarrow 0$.

Again by Lemma 3.1, the last 4 terms in (3.5) are bounded by $|x|^{k+1}$ times a constant independent of $a, b, x$. Thus, taking into account the result of the preceding paragraph, we see that $a / x^{k} \rightarrow(k-1) \delta$ as $(a, b, x) \in T$ and $x \rightarrow 0$.

Note that up to now we proved some properties of the solutions to (1.1), but we do not know yet whether those solutions exist, apart from $(0,0,0)$. We will establish it now. Recall that the order of contact is defined in such a way that the curves $y=0$ and $y=x^{n}$ have order of contact $n-1$.

Theorem 3.3. Consider the set $\Upsilon$ which is the projection to the $(a, b)$-plane of the set of solutions of the system (1.1), for $F$ of the form (3.2), under assumptions (1.4) and (3.3). Then, close to the origin, $\Upsilon$ is the union of two smooth curves, beginning at the origin, disjoint except at this point, and tangent there with the order of contact $1 /(k-1)$.

Proof. Recall that $k$ is an odd integer. We may assume that $-k \delta / \varepsilon>0$, using a coordinate change that replaces $b$ by $-b$ if necessary. Then by Lemma 3.2, close to the origin there are no solutions to (1.1) with $b<0$ (because then $b / x^{k-1}$ would be negative). For $b \geq 0$ we make a substitution $a=\widehat{a}^{k}, b=\widehat{b}^{k-1}$ and $x=\widehat{x} \widehat{b}$ in (3.5) and (3.6). We get

$$
\begin{aligned}
\widehat{G}(\widehat{a}, \widehat{b}, \widehat{x}) & =\widehat{a} \widehat{b}^{k}+\varepsilon \widehat{x} \widehat{b}^{k}+\delta \widehat{x}^{k} \widehat{b}^{k}+\widehat{b}^{k+1} \psi_{1}, \\
\widehat{H}(\widehat{a}, \widehat{b}, \widehat{x}) & =\varepsilon \widehat{b}^{k-1}+k \delta \widehat{x}^{k-1} \widehat{b}^{k-1}+\widehat{b}^{k} \psi_{2},
\end{aligned}
$$

where $\psi_{1}$ and $\psi_{2}$ are analytic (or sufficiently smooth) functions of $\widehat{a}, \widehat{b}, \widehat{x}$. For $b>0$ the system of equations $\widehat{G}=0, \widehat{H}=0$ is equivalent to (1.1). However, it is also equivalent to the system

$$
\left\{\begin{array}{l}
\widehat{a}+\varepsilon \widehat{x}+\delta \widehat{x}^{k}+\widehat{b} \psi_{1}=0, \\
\varepsilon+k \delta \widehat{x}^{k-1}+\widehat{b} \psi_{2}=0 .
\end{array}\right.
$$

For $\widehat{b}=0$ the system (3.9) becomes

$$
\left\{\begin{array}{l}
\widehat{a}+\varepsilon \widehat{x}+\delta \widehat{x}^{k}=0, \\
\varepsilon+k \delta \widehat{x}^{k-1}=0,
\end{array}\right.
$$


whose solutions (in $(\widehat{a}, \widehat{x}))$ are

$$
u_{-}=\left(-\frac{(k-1) \varepsilon}{k}\left(-\frac{\varepsilon}{k \delta}\right)^{\frac{1}{k-1}},\left(-\frac{\varepsilon}{k \delta}\right)^{\frac{1}{k-1}}\right)
$$

and

$$
u_{+}=\left(\frac{(k-1) \varepsilon}{k}\left(-\frac{\varepsilon}{k \delta}\right)^{\frac{1}{k-1}},-\left(-\frac{\varepsilon}{k \delta}\right)^{\frac{1}{k-1}}\right) .
$$

The gradients of the functions appearing in (3.10) (as functions of $\widehat{a}$ and $\widehat{x}$ ) at $u_{-}$and $u_{+}$are linearly independent. Therefore the solutions of (3.9) form two smooth curves transversally intersecting the plane $\widehat{b}=0$ at $u_{-}$and $u_{+}$. This takes care of all solutions of (3.9) sufficiently close to any given compact subset of the plane $\widehat{b}=0$. The only other solutions close to the plane $\widehat{b}=0$ that can exist have to escape to infinity in the $\widehat{a}, \widehat{x}$-directions. However, there are no such solutions, because by Lemma 3.2 in $T$, if $b \rightarrow 0$, then $\widehat{a}, \widehat{b}, \widehat{x}$ converge to finite limits.

For $b>0$ there is a one-to-one correspondence between the solutions of (1.1) and (3.9), and it is given by $a=\widehat{a}^{k}, b=\widehat{b}^{k-1}$ and $x=\widehat{x} \widehat{b}$. The solutions of (1.1) projected to the $(\widehat{a}, \widehat{b})$-plane are (close to the plane $\widehat{b}=0$ ) graphs of functions $\widehat{a}=g_{ \pm}(\widehat{b})$, with $g_{ \pm}(0)$ equal to the first component of $u_{ \pm}$. In the $(a, b)$-plane they are graphs of the functions $a=g_{ \pm}\left(b^{1 /(k-1)}\right) b^{k /(k-1)}$. Since $g_{-}(0) \neq g_{+}(0)$, this proves the properties of $\Upsilon$ stated in the theorem.

\section{Codimension 1}

In the codimension 1 case the integer $k$ in (1.2) is 3. Furthermore, the gradient of $A_{0}$ at the origin is non-zero (making a vector in $\mathbb{R}^{2}$ zero requires 2 equalities). Thus, we can perform the change of coordinates (3.1). The only non-generic hypothesis that we can make is that the gradients of $A_{0}$ and $A_{1}$ at the origin are linearly dependent. That is,

$$
\frac{\partial A_{0}}{\partial a}(0,0) \frac{\partial A_{1}}{\partial b}(0,0)-\frac{\partial A_{0}}{\partial b}(0,0) \frac{\partial A_{1}}{\partial a}(0,0)=0,
$$

which in view of (3.1) becomes

$$
\frac{\partial A_{1}}{\partial b}(0,0)=0
$$

Thus we can write

$$
F(a, b, x)=a+\beta b^{2} x+\gamma b x^{2}+\delta x^{3}+\varphi_{1} a x+\varphi_{2} b^{3} x+\varphi_{3} b^{2} x^{2}+\varphi_{4} b x^{3}+\varphi_{5} x^{4},
$$

$$
F_{x}(a, b, x)=\beta b^{2}+2 \gamma b x+3 \delta x^{2}+\varphi_{6} a+\varphi_{7} b^{3}+\varphi_{8} b^{2} x+\varphi_{9} b x^{2}+\varphi_{10} x^{3} .
$$

Note that $A_{3}(0,0)=\delta$, so in view of (1.4) and because $k=3$, we have

$$
\delta \neq 0 \text {. }
$$

Moreover, without changing the codimension, we can assume that

$$
\beta \neq 0 .
$$

Lemma 4.1. There exist constants $K_{1}, K_{2}, K_{3}, K_{4}$ such that $|b| \leq K_{1}|x|,|x| \leq$ $K_{2}|b|,|a| \leq K_{3}|b|^{3}$ and $|a| \leq K_{4}|x|^{3}$. 


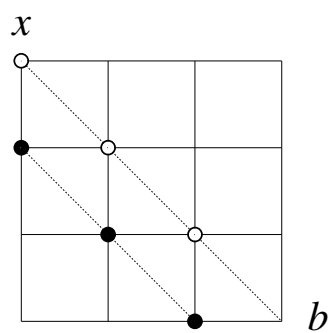

Figure 4. The lowest order non-zero coefficients for equations (4.3) and (4.4).

Proof. We claim that assumptions (0)-(5) are satisfied with $p=q=2$ and $t=\frac{3}{2}$. Indeed, $\mu(0,0,0)=1$, so (0) holds. Next, $\tau(b, x)=\beta b^{2}+2 \gamma b x+3 \delta x^{2}+\cdots$, so $c_{20}=\beta, c_{11}=2 \gamma, c_{02}=3 \delta$ and $c_{00}=c_{01}=c_{10}=0$; see the dots in Figure 4. Also $\sigma(b, x)=\beta b^{2} x+\gamma b x^{2}+\delta x^{3}+\cdots$, so, $d_{21}=\beta, d_{12}=\gamma, d_{03}=\delta$ and $d_{00}=d_{01}=d_{10}=d_{02}=d_{11}=d_{20}=d_{30}=0$; see the circles in Figure 4 (the only pairs $(i, j)$ for which $i / 2+j / 2<3 / 2$ are $(0,0),(0,1),(1,0),(0,2),(1,1)$ and $(2,0))$. This proves (1) and (2). Since $t \geq 1$, there are no $(l, i, j)$ satisfying the inequality in (3), so (3) holds. Since $t>1$, the sums in (4) and (5) are empty. Moreover, $c_{20}=\beta \neq 0$ and $c_{02}=3 \delta \neq 0$. This proves (4) and (5). Therefore, we can use Lemma 2.1. and we get the desired inequalities.

With the notation as in (4.3) and (4.4), set

$$
\Delta=\gamma^{2}-3 \beta \delta \text {. }
$$

Let $\zeta_{ \pm}$be the solutions to the equation $\beta t^{2}+2 \gamma t+3 \delta=0$ if $\Delta>0$ and let $\zeta$ be the only solution to this equation if $\Delta=0$.

Lemma 4.2. As $(a, b, x) \in T$ and $x \rightarrow 0$, if $\Delta>0$, then $b / x \rightarrow \zeta_{ \pm}$and $a / x^{3} \rightarrow$ $-\beta \zeta_{ \pm}^{2}-\gamma \zeta_{ \pm}-\delta$; if $\Delta<0$, then $(0,0,0)$ is an isolated point in $T$, and if $\Delta=0$, then $b / x \rightarrow \zeta$ and $a / x^{3} \rightarrow-\beta \zeta^{2}-\gamma \zeta-\delta$.

Proof. By Lemma 4.1 the last five terms in (4.4) divided by $x^{2}$ go to 0 when $x \rightarrow 0$ and $(a, b, x)$ stays in $T$. This proves that $b / x$ goes to solutions of the equation $\beta t^{2}+2 \gamma t+3 \delta=0$ as $(a, b, x) \in T$ and $x \rightarrow 0$. That is, if $\Delta>0$, then $b / x \rightarrow \zeta_{ \pm}$, and if $\Delta=0$, then $b / x \rightarrow \zeta$ (provided the solutions exist). Moreover, if $\Delta<0$ we get no solutions near the origin (except the origin itself).

By similar arguments, the last five terms in (4.3) divided by $x^{3}$ go to 0 when $x \rightarrow 0$ and $(a, b, x)$ stays in $T$. Thus, taking into account the result of the preceding paragraph we see that if $\Delta>0$, then $a / x^{3} \rightarrow-\beta \zeta_{ \pm}^{2}-\gamma \zeta_{ \pm}-\delta$, and if $\Delta=0$, then $a / x^{3} \rightarrow-\beta \zeta^{2}-\gamma \zeta-\delta$ as $(a, b, x) \in T$ and $x \rightarrow 0$.

We now investigate the existence of solutions.

Theorem 4.3. Consider the set $\Upsilon$ which is the projection to the $(a, b)$-plane of the set of solutions of the system (1.1) for $F$ of the form (3.2), under assumptions (1.4) with $k=3$, (4.1) and (4.6). If $\Delta>0$, close to the origin $\Upsilon$ is the union of two smooth curves, passing through the origin, disjoint except at this point, and tangent there with the order of contact 2 . If $\Delta<0$, then close to the origin $\Upsilon$ consists of a single point. 
Proof. Observe that if $(a, b, x) \in T$ is in a small neighborhood of the origin (but is not the origin), then by Lemma $4.2 b \neq 0$, because otherwise we would have $\zeta_{+}, \zeta_{-}$ or $\zeta$ equal to 0 . This would imply $\delta=0$, contrary to (4.5).

We make a substitution $a=\widehat{a} b^{3}$ and $x=\widehat{x} b$ in (4.3) and (4.4), and get

$$
\begin{aligned}
& \widehat{G}(\widehat{a}, b, \widehat{x})=\widehat{a} b^{3}+\beta \widehat{x} b^{3}+\gamma \widehat{x}^{2} b^{3}+\delta \widehat{x}^{3} b^{3}+\psi_{1} b^{4}, \\
& \widehat{H}(\widehat{a}, b, \widehat{x})=\beta b^{2}+2 \gamma \widehat{x} b^{2}+3 \delta \widehat{x}^{2} b^{2}+\psi_{2} b^{3},
\end{aligned}
$$

where $\psi_{1}$ and $\psi_{2}$ are analytic (or sufficiently smooth) functions of $\widehat{a}, b, \widehat{x}$. For $b \neq 0$ the system of equations $\widehat{G}=0, \widehat{H}=0$ is equivalent to (1.1). However, it is also equivalent to the system

$$
\left\{\begin{array}{l}
\widehat{a}+\beta \widehat{x}+\gamma \widehat{x}^{2}+\delta \widehat{x}^{3}+\psi_{1} b=0, \\
\beta+2 \gamma \widehat{x}+3 \delta \widehat{x}^{2}+\psi_{2} b=0
\end{array}\right.
$$

whose solutions (in $(\widehat{a}, \widehat{x}))$ for $b=0$ (in the limit case) are $u_{-}=\left(\widehat{a}_{-}, \widehat{x}_{-}\right)$and $u_{+}=\left(\widehat{a}_{+}, \widehat{x}_{+}\right)$, where

and

$$
\widehat{x}_{ \pm}=\frac{-\gamma \pm \sqrt{\Delta}}{3 \delta}
$$

$$
\widehat{a}_{ \pm}=-\left(\beta \widehat{x}_{ \pm}+\gamma \widehat{x}_{ \pm}^{2}+\delta \widehat{x}_{ \pm}^{3}\right) .
$$

Thus, two solutions exist if $\Delta>0$, and if $\Delta<0$ we get no solution.

We claim that if $\Delta>0$, then $\widehat{a}_{+} \neq \widehat{a}_{-}$. We have

$$
\begin{aligned}
\widehat{a}_{-}-\widehat{a}_{+} & =\beta\left(\widehat{x}_{+}-\widehat{x}_{-}\right)+\gamma\left(\widehat{x}_{+}^{2}-\widehat{x}_{-}^{2}\right)+\delta\left(\widehat{x}_{+}^{3}-\widehat{x}_{-}^{3}\right) \\
& =\left(\widehat{x}_{+}-\widehat{x}_{-}\right)\left(\beta+\gamma\left(\widehat{x}_{+}+\widehat{x}_{-}\right)+\delta\left(\widehat{x}_{+}^{2}+\widehat{x}_{+} \widehat{x}_{-}+\widehat{x}_{-}^{2}\right)\right) .
\end{aligned}
$$

Now,

and

$$
\widehat{x}_{+}+\widehat{x}_{-}=\frac{-2 \gamma}{3 \delta}
$$

Hence,

$$
\widehat{x}_{+}^{2}+\widehat{x}_{+} \widehat{x}_{-}+\widehat{x}_{-}^{2}=\left(\widehat{x}_{+}+\widehat{x}_{-}\right)^{2}-\widehat{x}_{+} \widehat{x}_{-}=\frac{4 \gamma^{2}}{9 \delta^{2}}-\frac{\beta}{3 \delta} .
$$

$\beta+\gamma\left(\widehat{x}_{+}+\widehat{x}_{-}\right)+\delta\left(\widehat{x}_{+}^{2}+\widehat{x}_{+} \widehat{x}_{-}+\widehat{x}_{-}^{2}\right)=\beta-\frac{2 \gamma^{2}}{3 \delta}+\frac{4 \gamma^{2}}{9 \delta}-\frac{\beta}{3}=\frac{6 \beta \delta-2 \gamma^{2}}{9 \delta}=\frac{-2 \Delta}{9 \delta}$.

Moreover,

so

$$
\widehat{x}_{+}-\widehat{x}_{-}=\frac{2 \sqrt{\Delta}}{3 \delta},
$$

$$
\widehat{a}_{-}-\widehat{a}_{+}=\frac{-4 \Delta \sqrt{\Delta}}{27 \delta^{2}} \neq 0 .
$$

If $\Delta>0$, then the gradients of the functions appearing in (4.10) (as functions of $\widehat{a}$ and $\widehat{x}$ ) with $b=0$, at $u_{-}$and $u_{+}$, are linearly independent. Thus, the three surfaces, two given by the equations (4.10) and the third one $b=0$, intersect transversally. Hence, the solutions to the system (4.10) form two smooth curves transversally intersecting the plane $b=0$. This takes care of all solutions of (4.10) sufficiently close to any given compact subset of the plane $b=0$. The only other solutions close to the plane $b=0$ that can exist have to escape to infinity in the $\widehat{a}, \widehat{x}$-directions. However, there are no such solutions by Lemma 4.2 and the fact that in $T$ if $b \rightarrow 0$, then $x \rightarrow 0$. 
Since $a=\widehat{a} b^{3}$, we see that close to the origin the set $\Upsilon$ consists of two curves of the form $\widehat{a}_{1}(b) b^{3}$ and $\widehat{a}_{2}(b) b^{3}$, where the limits of $\widehat{a}_{1}(b)$ and $\widehat{a}_{2}(b)$ as $b \rightarrow 0$ are $\widehat{a}_{+}$ and $\widehat{a}_{-}$, respectively. Since $\widehat{a}_{+} \neq \widehat{a}_{-}$, the order of contact of those two curves is 2 .

For $\Delta<0$ similar considerations show that the set of solutions of (4.10) close to the plane $b=0$ is empty.

\section{Codimension 2}

The codimension 2 case is more difficult, since we can have four distinct situations. We study each case separately.

If $k=5$ in (1.4) and the gradients of $A_{0}$ and of $A_{1}$ at the origin are non-zero and linearly independent, then Theorem 3.3 applies and the order of contact is $1 / 4$.

A codimension 2 case also takes place when $k=3$ in (1.4), the gradients of $A_{0}$ and of $A_{1}$ at the origin are non-zero and are linearly dependent, and

$$
\beta \neq 0 \text { and } \Delta=0 \text {. }
$$

Under these hypotheses (4.3) and (4.4) hold. Observe that by (4.5), (4.7) and (5.1) we have $\gamma \neq 0$. With the substitution $x=y-\beta b / \gamma$ and taking into account that $\Delta=0$, we have

$$
\begin{aligned}
a+\beta b^{2} x+\gamma b x^{2}+\delta x^{3} & =a+\frac{\gamma^{2}}{3 \beta} y^{3}-\frac{\beta^{2}}{3 \gamma} b^{3}, \\
\beta b^{2}+2 \gamma b x+3 \delta x^{2} & =\frac{\gamma^{2}}{\beta} y^{2} .
\end{aligned}
$$

Therefore this substitution applied to (4.3) and (4.4) gives

$$
\begin{aligned}
G(a, b, y) & =a-\frac{\beta^{2}}{3 \gamma} b^{3}+\frac{\gamma^{2}}{3 \beta} y^{3} \\
& +\xi_{1} a b+\xi_{2} a y+\xi_{3} b^{4}+\xi_{4} b^{3} y+\xi_{5} b^{2} y^{2}+\xi_{6} b y^{3}+\xi_{7} y^{4}, \\
H(a, b, y) & =\frac{\gamma^{2}}{\beta} y^{2}+\xi_{8} a+\xi_{9} b^{3}+\xi_{10} b^{2} y+\xi_{11} b y^{2}+\xi_{12} y^{3}
\end{aligned}
$$

where $\xi_{1}, \xi_{2}, \ldots$ are analytic (or sufficiently smooth) functions of the variables $a, b, y$ related to $\varphi_{1}, \varphi_{2}, \ldots$. Moreover, we did not assume any special properties or interdependencies of the functions $\varphi_{i}$ (separately in each equation), so it is easy to check using (5.2) and (5.3) that there are no such properties or interdependencies in (5.4) and (5.5), except that $\xi_{1}=-\varphi_{1} \beta / \gamma$ and $\xi_{2}=\varphi_{1}$, so $\xi_{1}=-\xi_{2} \beta / \gamma$.

Now we rewrite (5.4) and (5.5) separating the values of $\xi_{i}$ at the origin for $i=1,2,3,4,8,9$ :

$$
\begin{aligned}
G(a, b, y) & =a-\frac{\beta^{2}}{3 \gamma} b^{3}+\frac{\gamma^{2}}{3 \beta} y^{3}-\frac{\varkappa \beta}{\gamma} a b+\varkappa a y+\lambda b^{4}+\rho b^{3} y+\omega_{1} a^{2} \\
& +\omega_{2} a b^{2}+\omega_{3} a b y+\omega_{4} a y^{2}+\omega_{5} b^{2} y^{2}+\omega_{6} b y^{3}+\omega_{7} y^{4}+\omega_{8} b^{5}+\omega_{9} b^{4} y
\end{aligned}
$$

$$
\begin{aligned}
H(a, b, y) & =\frac{\gamma^{2}}{\beta} y^{2}+\varepsilon a+\zeta b^{3} \\
& +\omega_{10} a^{2}+\omega_{11} a b+\omega_{12} a y+\omega_{13} b^{2} y+\omega_{14} b y^{2}+\omega_{15} y^{3}+\omega_{16} b^{4}
\end{aligned}
$$

where $\omega_{i}$ are analytic (or sufficiently smooth) functions of the variables $a, b, y$. 
Set

$$
\vartheta=-\frac{\beta}{\gamma^{2}}\left(\frac{\varepsilon \beta^{2}}{3 \gamma}+\zeta\right) \text { and } \eta=\lambda-\frac{\varkappa \beta^{3}}{3 \gamma^{2}} .
$$

Without changing the codimension, we may assume that

$$
\zeta \neq 0 \text { and } \vartheta \neq 0 .
$$

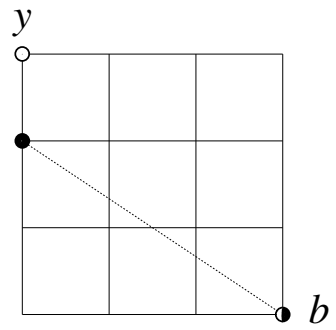

FiguRE 5. The lowest order non-zero coefficients for equations (5.6) and (5.7).

Lemma 5.1. There exist constants $K_{1}, K_{2}, K_{3}$ such that $|b|^{3} \leq K_{1} y^{2}, y^{2} \leq K_{2}|b|^{3}$ and $|a| \leq K_{3}|b|^{3}$.

Proof. In what we use from Section 2, we replace $x$ by $y$. We claim that assumptions (0)-(5) are satisfied with $p=2, q=3$ and $t=1$. Indeed,

$$
\mu(a, b, y)=1-\frac{\varkappa \beta}{\gamma} b+\varkappa y+\omega_{1} a+\omega_{2} b^{2}+\omega_{3} b y+\omega_{4} y^{2},
$$

so (0) holds. Further,

$$
\tau(b, y)=\frac{\gamma^{2}}{\beta} y^{2}+\zeta b^{3}+\omega_{13} b^{2} y+\omega_{14} b y^{2}+\omega_{15} y^{3}+\omega_{16} b^{4},
$$

so $c_{02}=\gamma^{2} / \beta \neq 0$ and $c_{30}=\zeta \neq 0$, while $c_{00}=c_{01}=c_{10}=c_{11}=c_{20}=0$; see the dots in Figure 5. This proves (1). Also,

$$
\sigma(b, y)=-\frac{\beta^{2}}{3 \gamma} b^{3}+\frac{\gamma^{2}}{3 \beta} y^{3}+\lambda b^{4}+\rho b^{3} y+\omega_{5} b^{2} y^{2}+\omega_{6} b y^{3}+\omega_{7} y^{4}+\omega_{8} b^{5}+\omega_{9} b^{4} y,
$$

so $d_{30}=-\beta^{2} /(3 \gamma), d_{03}=\gamma^{2} /(3 \beta)$ and $d_{00}=d_{01}=d_{10}=d_{11}=d_{20}=d_{02}=0$; see the circles in Figure 5. This proves (2). There are no $(l, i, j)$ satisfying $i / 3+j / 2+l<$ 0 , and this proves (3). The number $q t=3$ is an integer, and the only pair $(l, i)$ satisfying $i / 3+l=0$ is $(0,0)$. Thus, the condition from (4) is $-e_{000} d_{30}+c_{30} \neq 0$. Since

$$
\nu(a, b, y)=\varepsilon+\omega_{10} a+\omega_{11} b+\omega_{12} y,
$$

we have $e_{000}=\varepsilon$, so $-e_{000} d_{30}+c_{30}=-\vartheta \gamma^{2} / \beta$. Thus, by (5.8), (4) holds. Similarly, the condition from (5) is $-e_{000} d_{02}+c_{02} \neq 0$. Since $d_{02}=0$ and $c_{02} \neq 0$, this is true, so (5) holds. Thus, by Lemma 2.1, there exists constants $K_{1}, K_{2}, K_{3}$ such that $|b|^{3} \leq K_{1} y^{2}, y^{2} \leq K_{2}|b|^{3}$ and $|a| \leq K_{3}|b|^{3}$.

Lemma 5.2. As $(a, b, x) \in T, b \neq 0$ and $b \rightarrow 0$, then $a / b^{3} \rightarrow \beta^{2} /(3 \gamma)$ and $y^{2} / b^{3} \rightarrow \vartheta$. 
Proof. By Lemma [5.1, all terms of (5.6), except the first two, divided by $b^{3}$ go to 0 when $b \rightarrow 0$ and $(a, b, x)$ stays in $T$. This proves that $a / b^{3}$ goes to $\beta^{2} /(3 \gamma)$.

Similarly, all terms of (5.7), except the first three, divided by $b^{3}$ go to 0 when $b \rightarrow$ 0 and $(a, b, x)$ stays in $T$. Therefore, using the result of the preceding paragraph, we see that $y^{2} / b^{3}$ goes to $\vartheta$.

Lemma 5.3. Consider the set $\widetilde{\Upsilon}$ of solutions of the system (1.1) for $F$ of the form (3.2), under assumptions (1.4) with $k=3$, (5.1) and (5.8). Then close to the origin $\widetilde{\Upsilon}$ is the union of two smooth curves, beginning at the origin and disjoint except at this point.

Proof. By Lemma 5.1 if $(a, b, x) \in T$ and $b=0$, then also $y=a=0$. Now, for $b \neq 0$ we make a substitution

$$
a=s\left(\widehat{a}+\frac{\beta^{2}}{3 \gamma}\right) \widehat{b}^{6}, \quad b=s \widehat{b}^{2} \quad \text { and } \quad y=\widehat{y} \widehat{b}^{3},
$$

where $s=\operatorname{sgn} \vartheta$. This makes sense only when $s b>0$, but this is consistent with the last statement of Lemma 5.2. We get from (5.6) and (5.7)

$$
\begin{aligned}
\widehat{G}(\widehat{a}, \widehat{b}, \widehat{y})= & s \widehat{a} \widehat{b}^{6}+\frac{\gamma^{2}}{3 \beta} \widehat{y}^{3} \widehat{b}^{9}-\frac{\varkappa \beta}{\gamma}\left(\widehat{a}+\frac{\beta^{2}}{3 \gamma}\right) \widehat{b}^{8} \\
& +s \varkappa\left(\widehat{a}+\frac{\beta^{2}}{3 \gamma}\right) \widehat{y} \widehat{b}^{9}+\lambda \widehat{b}^{8}+s \rho \widehat{y} \widehat{b}^{9}+\psi_{1} \widehat{b}^{10}, \\
\widehat{H}(\widehat{a}, \widehat{b}, \widehat{y})= & \frac{\gamma^{2}}{\beta} \widehat{y}^{2} \widehat{b}^{6}+s \varepsilon\left(\widehat{a}+\frac{\beta^{2}}{3 \gamma}\right) \widehat{b}^{6}+s \widehat{b}^{6}+\psi_{2} \widehat{b}^{7},
\end{aligned}
$$

where $\psi_{1}$ and $\psi_{2}$ are analytic (or sufficiently smooth) functions of $\widehat{a}, \widehat{b}, \widehat{y}$.

For $\widehat{b} \neq 0$ the system of equations $\widehat{G}=0, \widehat{H}=0$ is equivalent to (1.1). However, it is also equivalent to the system

$$
\left\{\begin{array}{l}
s \widehat{a}+\eta \widehat{b}^{2}-\frac{\varkappa \beta}{\gamma} \widehat{a} \widehat{b}^{2}+\left(\frac{\gamma^{2}}{3 \beta} \widehat{y}^{2}+s \rho+\frac{s \varkappa \beta^{2}}{3 \gamma}\right) \widehat{y} \widehat{b}^{3}+s \varkappa \widehat{a} \widehat{y} \widehat{y}^{3}+\psi_{1} \widehat{b}^{4}=0, \\
\frac{\gamma^{2}}{\beta}\left(\widehat{y}^{2}-|\vartheta|\right)+s \varepsilon \widehat{a}+\psi_{2} \widehat{b}=0 .
\end{array}\right.
$$

For $\widehat{b}=0$ the system becomes

$$
\left\{\begin{array}{l}
\widehat{a}=0, \\
\frac{\gamma^{2}}{\beta}\left(\widehat{y}^{2}-|\vartheta|\right)+s \varepsilon \widehat{a}=0,
\end{array}\right.
$$

whose solutions (in $(\widehat{a}, \widehat{y}))$ are $u_{+}=(0, \sqrt{|\vartheta|})$ and $u_{-}=(0,-\sqrt{|\vartheta|})$. The gradients of the functions appearing in (5.10) (as functions of $\widehat{a}$ and $\widehat{y}$ ) at $u_{+}$and $u_{-}$are linearly independent. Therefore the solutions of (5.9) form two smooth curves transversally intersecting the plane $\widehat{b}=0$ at $u_{+}$and $u_{-}$. This takes care of all solutions of (5.9) sufficiently close to any given compact subset of the plane $\widehat{b}=0$. The only other solutions close to the plane $\widehat{b}=0$ that can exist have to escape to infinity in the $\widehat{a}, \widehat{y}$-directions. However, there are no such solutions, because by Lemma 5.2 in $T$, if $\widehat{b} \rightarrow 0$, then $\widehat{a}, \widehat{y}$ converge to finite limits.

We will now concentrate on the first equation of (5.9).

Lemma 5.4. There exists a constant $K_{4}$ such that $|\widehat{a}| \leq K_{4}|\widehat{b}|^{2}$. Moreover, $\widehat{a} / \widehat{b}^{2} \rightarrow$ $-s \eta$. 
Proof. We follow the proof of Lemma 2.1. If such a constant does not exist, then there is a sequence of points satisfying (5.9) for which $\widehat{b}_{n} \rightarrow 0$ and $\widehat{b}_{n}^{2} / \widehat{a}_{n} \rightarrow 0$. Dividing the first equation of (5.9) by $\widehat{a}_{n}$, we get the left hand side converging to $s$ as $n \rightarrow \infty$, while the right hand side stays 0 . This contradiction completes the proof of the first property.

Now, all terms of the first equation of (5.9), except the first two, divided by $\widehat{b}^{2}$ go to 0 , and this proves the second property.

Now we rewrite the first equation of (5.9) substituting $\widehat{a}=(\widehat{\widehat{a}}-s \eta) \widehat{b}^{2}$ and dividing it by $\widehat{b}^{2}$ (we still assume $\widehat{b} \neq 0$ ):

$$
s \widehat{\widehat{a}}+\left(\frac{\gamma^{2}}{3 \beta} \widehat{y}^{2}+s \rho+\frac{s \varkappa \beta^{2}}{3 \gamma}\right) \widehat{y} \widehat{b}+\psi_{3} \widehat{b}^{2}=0,
$$

where $\psi_{3}$ is an analytic (or sufficiently smooth) function of $\widehat{\widehat{a}}, \widehat{b}, \widehat{y}$.

Theorem 5.5. Consider the set $\Upsilon$ which is the projection to the $(a, b)$-plane of the set of solutions of the system (1.1) for $F$ of the form (3.2), under assumptions (1.4) with $k=3$, (5.1) and (5.8). Then close to the origin $\Upsilon$ is the union of two smooth curves, beginning at the origin, disjoint except at this point, and tangent there with the order of contact $7 / 2$.

Proof. The set $\Upsilon$ is the projection to the $(a, b)$-plane of the set $\widetilde{\Upsilon}$ from Lemma 5.3 . Thus, it remains to find out the order of contact of the two curves after the projection. By Lemma [5.2, $\widehat{y} \rightarrow \pm \sqrt{|\vartheta|}$ as $\widehat{b} \rightarrow 0$, where the sign is + for one curve and - for the other. Thus, by (5.11) and Lemma 5.4, the limit of $\widehat{\hat{a}} / \widehat{b}$ as $\widehat{b} \rightarrow 0$ is

$$
\mp \sqrt{|\vartheta|}\left(\frac{\gamma^{2}}{3 \beta} \widehat{y}^{2}+s \rho+\frac{s \varkappa \beta^{2}}{3 \gamma}\right)
$$

where again the sign depends on the curve. Thus, the difference of the values of $\widehat{\widehat{a}}$ on both curves, divided by $\widehat{b}$, goes to a non-zero constant. Looking at the substitutions that we made, we see that this difference divided by $\widehat{b}$ is equal to the difference of the values of $a$ divided by $\widehat{b}^{9}$, that is, by $(s b)^{9 / 2}$. Therefore in the $(a, b)$-plane the curves are tangent of order $7 / 2$.

\section{Another codimension 2 case}

Another codimension 2 phenomenon occurs when $k=3$ in (1.3) and the gradient of $A_{0}$ at the origin is zero. We may assume that the gradient of $A_{1}$ at the origin is non-zero. Therefore we can change (diffeomorphically) coordinates in the parameter plane and assume that $A_{1}(a, b)=a$. We can write

$$
\begin{gathered}
F(a, b, x)=\alpha b^{2}+a x+\varepsilon x^{3}+\varphi_{1} a x^{2}+\varphi_{2} b x^{2}+\varphi_{3} x^{4}+\varphi_{4} a^{2}+\varphi_{5} a b+\varphi_{6} b^{3} \\
F_{x}(a, b, x)=a+3 \varepsilon x^{2}+\varphi_{7} a x+\varphi_{8} b x+\varphi_{9} x^{3}
\end{gathered}
$$

Note that $A_{3}(0,0)=\varepsilon$, so in view of (1.4) and because $k=3$, we have

$$
\varepsilon \neq 0 \text {. }
$$

Moreover we can assume that

$$
\alpha \neq 0
$$




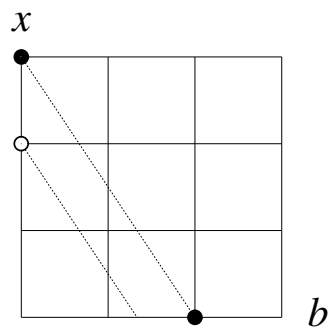

Figure 6. The lowest order non-zero coefficients for equations (6.2) and (6.1).

Lemma 6.1. There exist constants $K_{1}, K_{2}$ and $K_{3}$ such that $|b|^{2} \leq K_{1}|x|^{3},|x|^{3} \leq$ $K_{2}|b|^{2}$ and $|a| \leq K_{3}|x|^{2}$.

Proof. When using Lemma 2.1, we consider (6.2) to be the first equation and (6.1) to be the second one. We claim that assumptions (0)-(5) are satisfied with $p=3$, $q=2$ and $t=2 / 3$. Indeed $\mu(0,0,0)=1$, so (0) holds. Next, $\tau(b, x)=\alpha b^{2}+\varepsilon x^{3}+$ $\varphi_{2} b x^{2}+\varphi_{3} x^{4}+\varphi_{6} b^{3}$, so $c_{20}=\alpha, c_{03}=\varepsilon$ and $c_{00}=c_{01}=c_{10}=c_{11}=c_{02}=0$; see the dots in Figure 6. Also, $\sigma(b, x)=3 \varepsilon x^{2}+\varphi_{8} b x+\varphi_{9} x^{3}$, so $d_{02}=3 \varepsilon$ and $d_{00}=d_{01}=d_{10}=0$; see the circles in Figure 6. This proves (1) and (2). Now the only triple $(l, i, j)$ satisfying $i / 2+j / 3+2 l / 3<1 / 3$ is $(0,0,0)$, and we have $e_{000}=0$. This proves (3). The number $q t$ is not an integer, and we are done with (4). Furthermore, $p t=2$ is an integer, $d_{02}=3 \varepsilon, c_{03}=\varepsilon$, the only pair $(l, j)$ satisfying $j / 3+2 l / 3=1 / 3$ is the pair $(0,1)$ and $e_{001}=1$. This proves (5) because $-3 \varepsilon+\varepsilon=-2 \varepsilon \neq 0$. Thus, by Lemma 2.1, there exist constants $K_{1}, K_{2}$ and $K_{3}$ such that $|b|^{2} \leq K_{1}|x|^{3},|x|^{3} \leq K_{2}|b|^{2}$ and $|a| \leq K_{3}|x|^{2}$.

Lemma 6.2. As $(a, b, x) \in T$ and $x \rightarrow 0$, then $a / x^{2} \rightarrow-3 \varepsilon$ and $b^{2} / x^{3} \rightarrow 2 \varepsilon / \alpha$. Moreover, in the plane $x=0$ the origin is isolated in $T$.

Proof. By Lemma 6.1, the last 3 terms of (6.2) are bounded by $|x|^{5 / 2}$ times a constant independent of $a, b, x$. This proves that $a / x^{2} \rightarrow-3 \varepsilon$ as $(a, b, x) \in T$ and $x \rightarrow 0$.

Again by Lemma 6.1 the last 6 terms of (6.1) are bounded by $|x|^{7 / 2}$ times a constant independent of $a, b, x$. Thus, taking into account the result of the preceding paragraph, we see that $b^{2} / x^{3} \rightarrow 2 \varepsilon / \alpha$ as $(a, b, x) \in T$ and $x \rightarrow 0$.

Now consider what happens when $x=0$. By (6.2), $a=0$, so by (6.1) $\alpha b^{2}+$ $\varphi(b) b^{3}=0$, where $\varphi$ is an analytic (or sufficiently smooth) function of $b$. The solution $b=0$ is then isolated.

Theorem 6.3. Consider the set $\Upsilon$ which is the projection to the $(a, b)$-plane of the set of solutions of the system (1.1) for $F$ of the form (6.1), under assumptions (6.3) and (6.4). Then close to the origin $\Upsilon$ is the union of two smooth curves, beginning at the origin, disjoint except at this point, and not forming a cusp. More precisely, those curves are tangent at the origin, but go in opposite directions.

Proof. Set $s=\operatorname{sgn}(\alpha \varepsilon)$. By Lemma 6.2, if $x$ is close to 0 , then $b^{2} / x^{3}$ has the sign $s$, so since $b^{2}>0$, the sign of $x$ also has to be $s$. Thus, we consider only $x$ with $s x \geq 0$ and make the substitution $a=\widehat{a} \widehat{x}^{4}, b=\widehat{b} \widehat{x}^{3}$ and $x=s \widehat{x}^{2}$ in (6.1) and (6.2). Since 
we want this change of coordinates to be 1-to-1 (except that the whole plane $\widehat{x}=0$ corresponds to the origin in the $(a, b, x)$ coordinates), we consider only $\widehat{x} \geq 0$. We get

$$
\begin{aligned}
\widehat{G}(\widehat{a}, \widehat{b}, \widehat{x}) & =\alpha \widehat{b}^{2} \widehat{x}^{6}+s \widehat{a} \widehat{x}^{6}+s \varepsilon \widehat{x}^{6}+\psi_{1} \widehat{x}^{7} \\
\widehat{H}(\widehat{a}, \widehat{b}, \widehat{x}) & =\widehat{a} \widehat{x}^{4}+3 \varepsilon \widehat{x}^{4}+\psi_{2} \widehat{x}^{5}
\end{aligned}
$$

where $\psi_{1}$ and $\psi_{2}$ are analytic (or sufficiently smooth) functions of $\widehat{a}, \widehat{b}, \widehat{x}$.

For $s x>0$ the system of equations $\widehat{G}=0, \widehat{H}=0$ is equivalent to (1.1). However, it is also equivalent to the system

$$
\left\{\begin{array}{l}
\alpha \widehat{b}^{2}+s \widehat{a}+s \varepsilon+\psi_{1} \widehat{x}=0 \\
\widehat{a}+3 \varepsilon+\psi_{2} \widehat{x}=0
\end{array}\right.
$$

For $x=0$ the system becomes

$$
\left\{\begin{array}{l}
\alpha \widehat{b}^{2}+s \widehat{a}+s \varepsilon=0 \\
\widehat{a}+3 \varepsilon=0
\end{array}\right.
$$

whose solutions (in $(\widehat{a}, \widehat{b}))$ are

$$
u_{+}=\left(-3 \varepsilon, \sqrt{\left|\frac{2 \varepsilon}{\alpha}\right|}\right) \quad \text { and } \quad u_{+}=\left(-3 \varepsilon,-\sqrt{\left|\frac{2 \varepsilon}{\alpha}\right|}\right) .
$$

The gradients of the functions appearing in (6.5) (as functions of $\widehat{a}$ and $\widehat{b}$ ) at $u_{+}$ and $u_{-}$are linearly independent. Therefore the solutions of (6.5) form two smooth curves transversally intersecting the plane $\widehat{x}=0$ at $u_{+}$and $u_{-}$. This takes care of all solutions of (6.5) sufficiently close to any given compact subset of the plane $\widehat{x}=0$. The only other solutions close to the plane $\widehat{x}=0$ that can exist have to escape to infinity in the $\widehat{a}, \widehat{b}$-directions. However, there are no such solutions, because by Lemma 6.2 in $T$, if $\widehat{x} \rightarrow 0$, then $\widehat{a}, \widehat{b}$ converge to finite limits.

With $(a, b, x) \in T$ close to the origin, $\widehat{a}$ has to have the same sign as $-3 \varepsilon$, so $a=\widehat{a} \widehat{x}^{4}$ also has this sign. Similarly, $\widehat{b}$ has to be positive on the first curve and negative on the second one, so $b=\widehat{b} \widehat{x}^{3}$ is positive on the first curve and negative on the second.

Looking at the substitution we made, we see that $\widehat{x}=(b / \widehat{b})^{1 / 3}$, so $a=\left(\widehat{a} / \widehat{b}^{4 / 3}\right) b^{4 / 3}$. We have on $T$, as we approach the origin along each curve, $\widehat{a} / \widehat{b}^{4 / 3} \rightarrow-3 \varepsilon(\alpha /(2 \varepsilon))^{2 / 3}$ $\neq 0$ and $4 / 3>1$, so the projections to the $(a, b)$-plane of the two curves meet each other at the origin with tangent vectors in the opposite directions along the $b$-axis. This means that no cusp is formed, rather the union of the two curves is a curve of class $C^{1}$ (but not higher, because of the origin).

\section{Yet another CODIMENSION 2 CASE}

Yet another codimension 2 phenomenon occurs with assumptions such as those for the codimension 1 case (see Section 4), except (4.6). That is, we assume that $F$ is of the form (3.2), under assumptions (1.4) with $k=3$ and (4.1), but in the formulas (4.3) and (4.4) we set $\beta=0$. Thus, we get

$$
\begin{aligned}
F(a, b, x) & =a+\gamma b x^{2}+\delta x^{3}+\varphi_{1} a x+\varphi_{2} b^{3} x+\varphi_{3} b^{2} x^{2}+\varphi_{4} b x^{3}+\varphi_{5} x^{4}, \\
F_{x}(a, b, x) & =2 \gamma b x+3 \delta x^{2}+\varphi_{6} a+\varphi_{7} b^{3}+\varphi_{8} b^{2} x+\varphi_{9} b x^{2}+\varphi_{10} x^{3} .
\end{aligned}
$$


We can assume that

$$
\gamma \neq 0
$$

Note that those assumptions imply that $\Delta=\gamma^{2}>0$ (although we will not use this inequality explicitly).

If we try to use Lemma 2.1. we cannot assume that $\varphi_{7}(0,0,0)=0$ (this would give us codimension 3), so we get $p=2, q=3$, but $c_{11}=2 \gamma \neq 0$ (see Figure 7). Therefore assumption (1) of Lemma 2.1 is not satisfied. Fortunately, we do not need this lemma in its full extent, and we can prove what we need using a similar method.

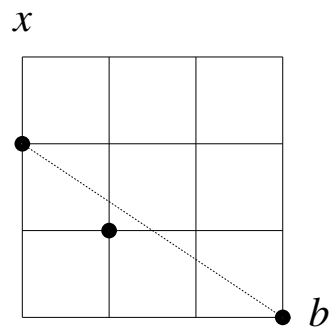

FiguRE 7. The lowest order non-zero coefficients for equation (7.2).

Lemma 7.1. There exist constants $K_{1}$ and $K_{2}$ such that $|x| \leq K_{1}|b|$ and $|a| \leq$ $K_{2}|b|^{3}$.

Proof. Suppose that there is no constant $K_{1}$ such that $|x| \leq K_{1}|b|$. Then there exists a sequence of points $\left(a_{n}, b_{n}, x_{n}\right) \in T$ convergent to $(0,0,0)$ such that $b_{n} / x_{n} \rightarrow$ 0 . By dividing (7.1) by $x^{3}$ and setting $z=b / x$, we get

$$
\frac{a}{x^{3}}+\gamma z+\delta+\psi_{1} \frac{a}{x^{3}} x+\psi_{2} x=0
$$

where $\psi_{1}$ and $\psi_{2}$ are analytic (or sufficiently smooth) functions of $a, x, z$. Therefore $a_{n} / x_{n}^{3} \rightarrow-\delta$ as $n \rightarrow \infty$.

Using the same substitution in (7.2) divided by $x^{2}$, we get

$$
2 \gamma z+3 \delta+\psi_{3} \frac{a}{x^{3}} x+\psi_{4} x=0
$$

where $\psi_{3}$ and $\psi_{4}$ are analytic (or sufficiently smooth) functions of $a, x, z$. Taking into account the result of the preceding paragraph, we get $\delta=0$ by taking a limit along our sequence, a contradiction. This completes the proof of the first inequality of the lemma.

Now, if $b=0$, then by what we already proved $x=0$, so by (17.1), $a=0$. If $b \neq 0$, then we divide (7.1) by $b^{3}$, and we get, setting $y=x / b$,

$$
\frac{a}{b^{3}}+\gamma y^{2}+\delta y^{3}+\psi_{5} \frac{a}{b^{3}} b y+\psi_{6} b y=0,
$$

where $\psi_{5}$ and $\psi_{6}$ are analytic (or sufficiently smooth) functions of $a, b, y$. As $a, b \rightarrow$ $0, y$ stays bounded (as we proved), so $a / b^{3}$ stays bounded.

Lemma 7.2. As $(a, b, x) \in T$ and $b \rightarrow 0$, then either $x / b \rightarrow 0$ and $a / b^{3} \rightarrow 0$ or $x / b \rightarrow-2 \gamma /(3 \delta)$ and $a / b^{3} \rightarrow-4 \gamma^{3} /\left(27 \delta^{2}\right)$. 
Proof. By Lemma 7.1, all but the first two terms of (7.2) divided by $b^{2}$ go to 0 when $b \rightarrow 0$ and $(a, b, x)$ stays in $T$. This proves that $x / b$ goes to the solutions of the equation $2 \gamma t+3 \delta t^{2}=0$. Those solutions are 0 and $-2 \gamma /(3 \delta)$.

By similar arguments, all but the first three terms of (7.1) divided by $b^{3}$ go to 0 , so we get $a / b^{3} \rightarrow-\gamma t^{2}-\delta t^{3}$, where $t$ is the solution from the preceding paragraph. Thus, we get as the limit of $a / b^{3}$ the values 0 in the first case and

$$
-\gamma\left(\frac{-2 \gamma}{3 \delta}\right)^{2}-\delta\left(\frac{-2 \gamma}{3 \delta}\right)^{3}=-\frac{4 \gamma^{3}}{27 \delta^{2}}
$$

in the second case.

Theorem 7.3. Consider the set $\Upsilon$ which is the projection to the $(a, b)$-plane of the set of solutions of the system (1.1) for $F$ of the form (7.1), under the assumptions (7.3). Then close to the origin $\Upsilon$ is the union of two smooth curves, passing through the origin, disjoint except at this point, and tangent there with the order of contact 2 .

Proof. We make a substitution $x=\widehat{x} b$ and $a=\widehat{a} b^{3}$ in (7.1) and (7.2). We get

$$
\begin{aligned}
& G(\widehat{a}, b, \widehat{x})=\widehat{a} b^{3}+\gamma \widehat{x}^{2} b^{3}+\delta \widehat{x}^{3} b^{3}+\xi_{1} b^{4}, \\
& H(\widehat{a}, b, \widehat{x})=2 \gamma \widehat{x} b^{2}+3 \delta \widehat{x}^{2} b^{2}+\xi_{2} b^{3},
\end{aligned}
$$

where $\xi_{1}, \xi_{2}$ are analytic (or sufficiently smooth) functions of $\widehat{a}, b, \widehat{x}$. For $b \neq 0$ the system of equations $G=0, H=0$ is equivalent to (1.1). However, it is also equivalent to the system

$$
\left\{\begin{array}{l}
\widehat{a}+\gamma \widehat{x}^{2}+\delta \widehat{x}^{3}+\xi_{1} b=0, \\
2 \gamma \widehat{x}+3 \delta \widehat{x}^{2}+\xi_{2} b=0 .
\end{array}\right.
$$

For $b=0$ the system (7.6) becomes

$$
\left\{\begin{array}{l}
\widehat{a}+\gamma \widehat{x}^{2}+\delta \widehat{x}^{3}=0, \\
2 \gamma \widehat{x}+3 \delta \widehat{x}^{2}=0,
\end{array}\right.
$$

whose solutions (in $(\widehat{a}, \widehat{x}))$ are

$$
u_{0}=(0,0) \quad \text { and } \quad u_{1}=\left(-\frac{4 \gamma^{3}}{27 \delta^{2}}, \frac{-2 \gamma}{3 \delta}\right) .
$$

The gradients of the functions appearing in (7.7) (as functions of $\widehat{a}$ and $\widehat{x}$ ) at $u_{0}$ and $u_{1}$ are linearly independent. Therefore the solutions of (7.6) form two smooth curves intersecting transversally the plane $b=0$ at $u_{0}$ and $u_{1}$. This takes care of all solutions of (7.6) sufficiently close to any given compact subset of the plane $b=0$. The only other solutions close to the plane $b=0$ that can exist have to escape to infinity in the $\widehat{a}, \widehat{x}$-directions. However, there are no such solutions, because by Lemma 7.2 in $T$, if $b \rightarrow 0$, then $\widehat{a}, \widehat{x}$ converge to finite limits. Finally, if $b=0$, then by Lemma 7.1 we also have $a=x=0$.

Thus, close to the origin $\Upsilon$ is the union of two smooth curves, passing through the origin, disjoint except at this point, and tangent there with the order of contact 2 (because we had $a=\widehat{a} b^{3}$ ). 


\section{REFERENCES}

[1] V. I. Arnold, Small denominators I, Mappings of the Circumference onto Itself, Amer. Math. Soc. Translations 46, 213-284 (1965).

[2] H. W. Broer, M. Golubitsky and G. Vegter, The geometry of resonance tongues: A singularity theory approach, Nonlinearity 16, 1511-1538 (2003). MR.1986309(2004c:37104)

[3] H. Broer and C. Simó, Resonance tongues in Hill's equations: A geometric approach, J. Diff. Equations 166, 290-327 (2000). MR1781258 (2001h:70036)

[4] H. Broer and C. Simó, Hill's equation with quasi-periodic forcing: Resonance tongues, instability pockets and global phenomena, Bul. Soc. Bras. Mat. 29, 253-293 (1998). MR1654831 (99m:34109)

[5] H. Broer, J. Puig, and C. Simó, Resonance tongues and instability pockets in the quasiperiodic Hill-Schrödinger equation, Commun. Math. Phys. 241, 467-503 (2003). MR2013807 $(2004 \mathrm{j}: 37028)$

[6] H. Broer, C. Simó, and J. C. Tatjer, Towards global models near homoclinic tangencies of dissipative diffeomorphisms, Nonlinearity 11, 667-770 (1998). MR 1617974 (2000g:37060)

[7] J. K. Hale and H. Koçak, "Dynamics and bifurcations," Texts Appl. Math. 3, Springer, New York (1991). MR.1138981 (93e:58047)

[8] M. Misiurewicz and A. Rodrigues, Double Standard Maps, Commun. Math. Phys. 273, 37-65 (2007). MR2308749 (2008b:37067)

[9] M. Misiurewicz and A. Rodrigues, On the tip of the tongue, J. of Fixed Point Theory Appl. 3, 131-141 (2008). MR2402913

[10] J. Puig and C. Simó, Analytic families of reducible linear quasi-periodic differential equations, Ergodic Th. and Dynam. Sys. 26, 481-524 (2006). MR2218772(2007b:37140)

Department of Mathematical Sciences, Indiana University-Purdue University Indianapolis, 402 N. BlackFord Street, Indianapolis, Indiana 46202-3216 - And - Institute of Mathematics of the Polish Academy of Sciences, Śniadeckich 8, 00-956 Warszawa, POLAND

E-mail address: mmisiure@math.iupui.edu

Department of Mathematical Sciences, Indiana University-Purdue University Indianapolis, 402 N. Blackford Street, Indianapolis, Indiana 46202-3216 - AND - CMUP, Rua do Campo Alegre 687, 4169-007 Porto, Portugal

E-mail address: arodrig@math.iupui.edu

Current address: Matematiska Institutionen, KTH, SE-100 44 Stockholm, Sweden

E-mail address: amdsar@kth.se 